\title{
Die Bildung von Bogenfasern als primäre Reflexbahn der vitalen (protopathischen) Empfindungen.
}

VON

\author{
G. U. ARIËNS KAPPERS (AMsTERDAM).
}

Man nimmt im allgemeinen und aus guten Gründen an, dasz die ersten Reize, für welche die Haut empfindlich ist, die sogenannten vitalen Reize sind - d.h. solche, welche das Leben. und Verhalten des Tieres beeinflussen.

Diese Reize, welche von HEaD deshalb auch wohl als protopathische Reize bezeichnet wurden (von protos $=$ erst entstandene und pathein $=$ fühlen), verlaufen, im Nervensystem angelangt, auf gekreuzten (Bogen-)Fasern zu der anderen Seite hinüber und werden dort also auf die andere Seite'des Körpers - effektuiert oder nach oben geleitet.

Neben diesen vitalen oder protopathischen Reizen entsteht mehr und mehr eine Empfindlichkeit für Reize, welche von HEad als epikritische Reize bezeichnet wurden — die man auch gnostische Reize nennen könnte. Es sind dies feinere (mehr kritische) Empfindungen, welche den. Körper, das Leben selber, nicht direkt beeinflussen, denen gegenüber das Leben relativ indifferent steht.

Diese Reiz-Empfindlichkeit bildet sich erst später aus, und zwar im Anschlusse an vitale Reize und deren Organe.

Head hat diese Einteilung nur für Hautempfindungen aufgestellt. Meines Erachtens gilt sie aber auch für die Empfindungen der höheren Sinnesorgane:

Der vitale Faktor in den Sinnesemptindungen des Labyrinthes ist der Gleichgewichtsnerv, der N. vestibularis. Seine Empfindungen sind für die Haltung des Körpers, für die Gravitätsstatik (kurz Gravistatik) von direkter Bedeutung. Sie werden sofort effektuiert, und steigen nicht oder kaum zum Bewusztsein auf (werden gnostisch kaum verwertet).

Die epikritische oder gnostische Empfindung der Sinnesorgane des Labyrinthes ist das Gehör, vom N. cochlearis übermittelt, dessen Empfindungen für das Verhalten oder für die Haltung des Körpers meistens nur von wenig Gewicht sind, gnostisch aber (beim Menschen z. B.) höchste Bedeutung haben.

. So hat auch das Auge vitale Komponenten. Die primitive Lichtempfindung, welche für die Photostatik wichtig ist, reguliert den Stand des Körpers in Bezug auf die Lichtquelle, auf das Licht im allgemeinen, und ist für das Leben - namentlich bei niederen Tieren, die für ihren Körper und ihre Nahrungssuche gewisser Lichtverhältnisse bedürfen, von groszer Bedeutung.

Der gnostische Faktor des Lichtes, für die Erkenntnis der Welt (auch beim Lesen und Schreiben u. s. w.) ist ohne weiteres deutlich. Letztere wird nicht unmittelbar effektuiert aber, ins Bewusztsein aufgenommen, trägt sie bei zu unserem Weltbild.

Der vitale Faktor der Hautempfindungen ist die Wahrnehmung der groben Berührungen, der gröszeren Kälte oder Wärme und des Schmerzes.

Hierauf wird wieder reflektorisch geantwortet, meistens durch Abwehrbewegungen.

Die vitalen Hautempfindungen dringen zwar ins Bewusztsein durch, namentlich der Schmerz, sie werden aber bald vermieden und zunächst nicht weiter gesucht, untersucht, d. h. nicht gnostisch verwertet. 
Die gnostischen Empfindungen des Körpers, der Haut (und der Muskelsensibilität) ermitteln dagegen unsere Stereognosis, d. h. unsere Kenntnis des Raumes und der Objekte in dem Raume, insofern dieselben durch Betastung erkannt werden können (man denke an den Blinden).

Auf diese feinen, gnostischen Reize wird nicht notwendigerweise unmittelbar reflektorisch geantwortet. Sie werden auch nicht vermieden, im Gegenteil aufgesucht.

So viel über das, was die Unterscheidung in vitale und gnostische Reize aubelangt.

In zweiter Instanz möchte ich darauf hinweisen, dasz die motorische Effektuierung, welche auf diese beidên Reizkategorien folgt, meistens sehr verschieden ist. Abgesehen noch davon, dasz vitale Reize mehr reflektorisch effektuiert werden als gnostische Reize, ist es auch eine bekannte Tatsache, dasz die motorische Antwort auf protopathische Hautreize meistens negativ ist, d. h. der Reiz wird vermieden. Dies gilt m. E. auch für vitale Sinnesempfindungen:

Die stark einseitige Empfindung einer Macula des Gleichgewichtsorganes wird vermieden, besser : korrigiert durch eine solche Veränderung der Körperstellung, dasz der Reiz wieder ins Gleichgewicht kommt mit anderen Reizen.

Die zu starke Lichtaufnahme, namentlich, wenn sie einseitig ist, wird korrigiert, vermieden. Viele photostatische Tiere: Fische, z. B. fliehen das starke Licht und leben im Halbdunkel.

Namentlich aber bei den Hautempfindungen ist die Negativität der vitalen oder protopathischen Reflexe klar (Sherrington).

Starke Kälte oder Wärme, starke Temperaturschwankungen im allgemeinen, Schmerz, grobe Berührungen: auf alle diese Reize wird fast immer geantwortet mit negativen Reflexbewegungen, d. h. mit einem Entfliehen.

Dem steht gegenüber, dasz epikritische, gnostische Reize oft positive Reflexe zum Vorschein rufen. Man neigt sich dem Flüstern zu, den Lauten - wenn sie nicht durch ihre Härte in die Reihe der protopathischen fallen.

Das Auge sucht sich zu orientieren über das Wesen der Dinge, das es lockt, solange nicht die Härte des Lichtes abstöszt. Gerade ein sanft schimmerndes Licht reizt unsere Aufmerksamkeit, unsere Zuneigung.

Die Hand, die Fingerspitzen suchen die Erfahrung der tastbaren Wirklichkeit, wenn sie nicht schmerzt.

Den zwei Kategorien der Empfindungen entsprechen also im groszen und ganzen zwei Kategorien von Reflexen.

Weniger bekannt ist es, dasz ihnen auch zwei Kategorien von Fasersystemen im Zentralnervensystem entsprechen.

Namentlich für die Hautempfindungen ist dies in meisterhafter Weise ermittelt worden.

Unter den Untersuchern, welche sich dabei ein Verdienst erworben haben, nenne ich nur Edinger, Fabritius, Petrìn und Brouwer.

Wir wissen, dasz sich die vitalen Reize im Rückenmarke fast sofort kreuzen, dasz dagegen die gnostischen Reize auf derselben Seite aufsteigen und absteigen, und sich auch in ihrem spinalen sensitivo-motorischen Reflex-Organismus nicht oder kaum kreuzen.

Die Auffassung, dasz die aufsteigenden sensiblen Wurzelfasern blosz dazu dienen, mittelst der Hinterstrangkerne und Thalamus die gnostischen Eindrücke auf das Vorderhirn zu projizieren, ist nicht richtig. Manche geben auch Kollateralen ab in die motorische Region des Rückenmarkes (überwiegend gleichseitig). Bei niederen Tieren ist dies sogar ihre Hauptfunktion. Bei der absteigenden Dichotomie jener Fasern ist dies überall die Hauptfunktion.

Eine Erklärung für dieses Verhalten ist indessen nie gegeben, sogar nie versucht worden. Weshalb zieht der vitale Reiz fast sofort auf die andere Seite des Rückenmarkes hinüber?

Weshalb führen die gleichseitig auf- und absteigenden Hinterwurzeldichotomien und deren überwiegend gleichseitige Kollateralen die der gnostischen Empfindungen?

Weshalb ist es nicht gerade umgekehrt im Rückenmark?

Eine vollständige Erklärung, auf Grund der in den letzten zehn Jahren ermittelten Gesetze der Neurobiotaxis und stimulogenen Fibrillation kann ich jetzt noch nicht geben, obschon einige Punkte uns auch jetzt schon dafür den Weg weisen.

Ich möchte hier zunächst hinweisen auf den Verband, der besteht zwischen diesen ver- 
schiedenen Kategorien von Empfindungen und deren verschiedenartigen Reflexen einerseits und dem eigentümlichen Verlaufe ihrer Rückenmarkswege anderseits.

Betrachten wir in Fig. 1 den Kreis $\mathrm{R}$ als eine nicht funktionierende Reizquelle, in Fig. 2 den Kreis $R^{\prime}$ als Quelle starker protopathischer (vitaler) Reize, in Fig. $3 R^{\prime \prime}$ als Quelle

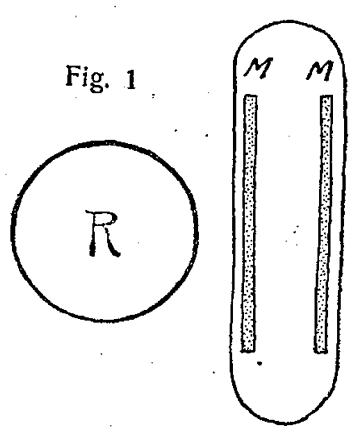

Fig. 2
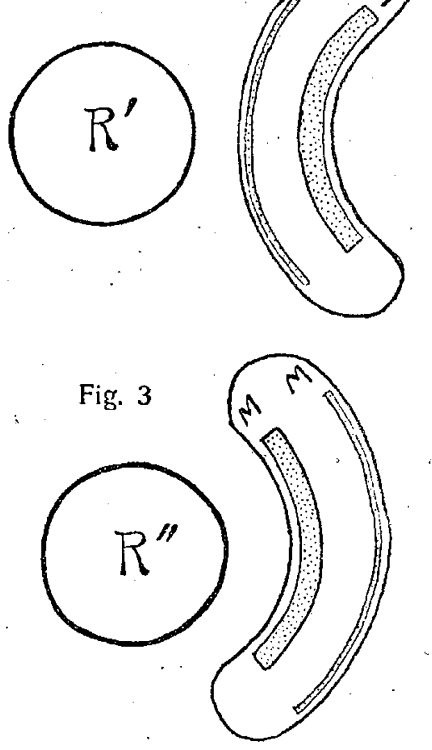

$\mathrm{R}=$ Reizquelle

Fig. 2 demonstriert die Abneigun un der Reizquelle bei Kontraktion de Kontrolateralen, Fig. 3 die Zuneigung bei Kontraiktion der gleichseitigen Muskelı (M.) der (meistens weniger intensiven) gnostischen (epikritischen) Reize, dann sehen wir dasz eine Abneigung von den vitalen Reizen in Fig. 2 erreicht wird durch Kontraktion der von $R$ abgekehrten Längsmuskulatur (M), während in Fig. 3 die Zuneigung zu dem gnostischen Reiz zu stande kommt durch eine Kontraktion der $R^{\prime \prime}$ zugekehrten Längsmuskulatur (M).

Die gekreuzte Übertragung des Reizstromes in dem Rückenmarke im ersteren Falle ist also in völliger Übereinstimmung mit dem negativen Reflex, d. h. mit der Abneigung von der Reizquelle, während im zweiten Falle der positive Reflex, (die Zuneigung zu der Reizquelle) und die gleichseitige Reflexübertragung mit einander in Übereinstimmung sind.

Figur 4 zeigt uns auf einem Querschnitt den Verlauf des Reflexreizes bei negativer Antwort auf vitalen Reflexwegen, Fig. 5 bei positiver Antwort auf gnostische Reize.

Im exsteren Falle findet man einen gekreuzten, im zweiten einen homolateralen Verlauf und Ablauf des Reizes.

Auch der Nutzen des gleichseitigen Aufsteigens und Absteigens der dichotomisierten Fasern ist unter diesem Gesichtspunkt deutlich, weil dadurch die nahe liegenden gleichseitigen Segmente dieselbe Wirkung integrieren.

Wie jedoch gesagt ist, möge die Sache uns teleologisch hierdurch näher gerückt sein, die Ursache jener Bahnbildung ist damit nicht erkannt.

Diese müssen wir in den Gesetzen finden, welche uns die Reize haben kennen lernen als die gestaltenden Kräfte des Nervensystems.

Die Frage, die uns jetzt interessiert, ist also diese: wie kann man sich denken, dasz die gestaltenden Kräfte des Nervensystems diese Fasern gebildet haben?

In dieser Hinsicht verweise ich auf die von mir an anderen Stellen öfters erwähnten Gesetze der Neurobiotaxis und diejenigen der stimulogenen Fibrillation, welche in sehr ingeniöser Weise von BoK ans Licht gebracht wurden.

Die Gesetze der Neurobiotaxis können kurz

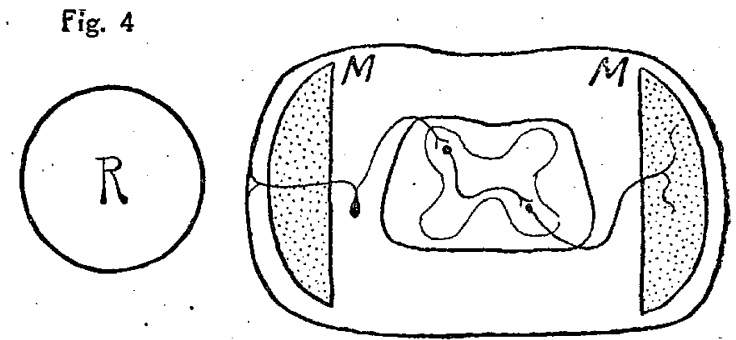

Fig, 5

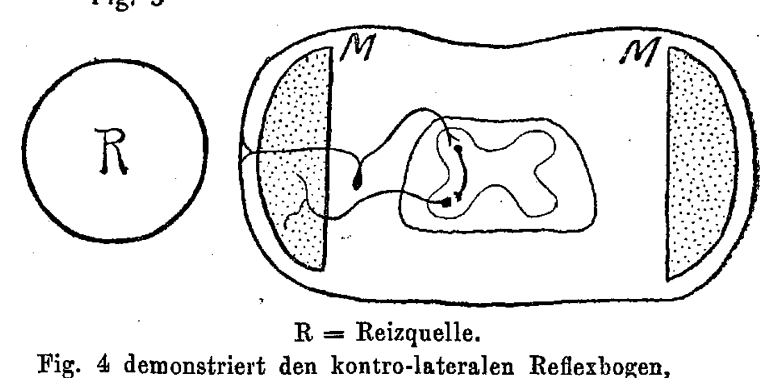

Fig. 4 demonstriert den kontro-lateralen Reflexbogen, Fig. I den humo-lateralen Reflexbogen der Hautsensibilität. zusammengefaszt werden in den Satz: dasz Bahnverbindungen, seien es dendritische, seien es axonale, sich immer bilden zwischen stimulativ korrelierten Gebieten, und dasz dabei die Haupt-Dendriten sich bilden und schlieszlich auch der Zelleib der Ganglienzelle sich verschiebt in der Richtung de maximalen Reizladung.

Das Gesetz der stimulogenen Fibrillation fügt dem hinzu, dasz die Achsenzylinder (die sich ebenfalls zwischen stimulativ korrelierten Gebieten bilden) auswachsten mit dem Reizverlauf, 
dasz sie im Gegensatz zu den Dendriten (die stimulo-petal wachsen), stimulo-fugal oder, besser gesagt, stimulo-konkurrent (mit dem Reiz mitgehend) wachsen, und zwar (wie bekannt) vor der Bildung der Dendriten.

Diese beiden Gesetze hat Bok neuerdings benutzt, die Bildung und die bestimmte Selektivität in der Ausbildung der motorischen Wurzelverbindungen der Reflex-Bahnen im allgemeinen zu erklären.

In welcher Weise diese Gesetzmäszigkeiten nun auf die Ausbildung der positiven und negativen Reflexe und deren Bahnen bei verschiedenartigen Reizungen derselben Hautstelle angewandt werden können, erfordert noch weitere Untersuchungen.

Indessen haben wir in der Mitteilung Bors schon wertvolle Hinweise für die Erklärung des gekreuzten Verlaufes der primitiven sekundären Sensibilitätsbahn. Bok hat in $191 \mathrm{y}$ darauf hingewiesen, dasz der Reflexbogen veranlaszt wird von dem Effektor, in dem Sinne, dasz dieser alle Reize zu sich zieht, welche infolge seiner Kontraktion , den Körper treffen.

Es ist nun klar, dasz, wenn ein Tier (wie das obengezeichnete) in einem an allen Seiten gleichmäszig reizenden Medium liegt (also ohne eine besonders lokalisierte Reizquelle, wie in der Figur) eine, durch irgend einen anderen Einflusz verursachte Kontraktion des linken Muskels die rechte Seite des Körpers nach auszen exponiert, d.i. mehreren und stärkeren Einflüssen aussetzt, als die gebeugte Seite.

Die gestreckte Seite wird infolgedessen diejenige, welche die meisten Reize von der Auszenwelt empfängt, und diese Reize werden sich nach den Bokschen Regeln der Reflexbahnbildung korrelieren mit der kontrolateralen Muskelkontraktion.

Ueber den scheinbaren Widerspruch dieser Genese der Reflexbahn mit den Erfahrungen von Uexkiull und Magnus, dasz im ausgebildeten Nervensystem ein Reiz geneigt ist in einen erschlafften Muskel hinein zu ziehen, darüber später ${ }^{1}$ )

In Übereinstimmung mit meiner Auseinandersetzung ist es, dasz die. Ausbildung der Bogenfasern, welche (vergl. Fig. 4) den negativen Reflex ermitteln ${ }^{2}$ ), in der Phylogenese der gleichseitigen Reizübertragung und den positiven Reflexen, namentlich der Ausbildung der gleichseitigen sensitivo-motorischen Reflexkollateralen ${ }^{3}$ ) voran geht.

Hiermit in Übereinstimmung ist auch, dasz das Hochhinaufsteigen der gleichseitigen Wurzelfasern erst predominant wird bei höheren Tieren, und dasz es gerade diese Fasern sind, welche mittelst der Hinterstrangkerne und des Zwischenhirns eine Projektion erhalten auf der Rinde, welche bekanntlich das gnostische Zentrum kat' exogen darstellt.

1) Hier sei blosz bemerkt, dasz nach Magnus diese Reflexauslösung bedingt wird durch die Anwesenheit der sensiblen Innervation in der erschlafften Extremität. Dasz hier ein erschlaffter Muskel-Tonus den Reizstrom anzieht, glaube ich nicht, vielmehr ein Zustand von Reizung jener Gegend durch Reckung. Jedenfalls liegt dabei auch ein den Gesetzen der Neurobiotaxis entsprechendes Korrelat vor.

2) Wie auch der Abwehrreflex des Fuszes und del 'Zehen (BABrssrx) ontogenetisch älter ist, als die Zuneigung jener Teile zum Fuszsohlreiz.

3) Dieselben entstehen erst beim Frosch (von L̇enhossek). 


\section{I T E R A T UR.}

EDINGER, Einiges vom Verlauf der Gefühlsbahnen. Deutsche mediz. Wochenschr. 1890.

Pètren, Ein Beitrag zur Frage vom Verlaufe der Bahnen vom Hautsinne u.s. w. Scandin. Arch. f. Physiol. Bnd. 13, 1902. SherRington, The integrative action of the nervous system. London, 1906.

HEAD and Rivers, A human experiment in Nerve division. Brain Fol. 31, 1908.

Fabritius, Studien über die sensible Leitung im menschlichen Rückenmark u.s. w. Arbeiten a. d. path. Instit. der Universität Helsingfors. Bnd. II, 1908.

ARIËNS KAPPERS, Weitere Mitteilungen über Neurobiotaxis̀. Die Selektivität der Zellenwanderung. Die Bedeutung synchronischer Reizverwandschaft. Folia Neurobiologica. Bnd. I, 1908.

Bok, Die Entwicklung der Hirnnerven und ihrer zentralen Bahnen. Die Stimulogene Fibrillation. Folia Neurobiologica. Bnd. IX, 1915

Brouwer, Die biologische Bedeutung der Dermatomerie. Folia Neurol. Bnd. 9, 1915.

ARIËNS KaPPERS, Further Contributions on Neurobiotaxis No. IX. The dynamic polarisation of the Neurone. Journ. of Comp. Neur. Bnd. 27, 1917. - Psych. en Neur. Bladen, Amsterdam, 1916.

Bok, The development of reflexes and reflextracts I. The reflex-circle. Psych. en Neur. Bladen, Amsterdam, 1917. 